\title{
Coronavirus Disease (COVID-19)/SARS-CoV-2: Hopefully, the Human-Virus Battle Ends Soon on a Positive Note
}

\author{
Venkataramana Kandi \\ *Corresponding author \\ Dr. Venkataramana Kandi Associate Professor of Microbiology, Prathima Institute of Medical Sciences, \\ Karimnagar, Telangana, India
}

ramana_20021@rediffmail.com,ramana20021@gmail.com

Abstract

The emergence and re-emergence of infectious diseases are not uncommon to humans. We have been seeing this, repeated many a time in the past, that the new/novel microbial species emerge and pose a potential threat to the whole of mankind. Among those infectious diseases which threatened mankind, the Smallpox virus appears to have had the greatest impact. Smallpox virus was suspected to be present on the earth since 10,000 B.C., but its presence and the effect on humans was established only in the late 18th century to the early 20th century when people suffered from its consequences. Most people (>75\%) infected with smallpox died, leaving a sense of doom among humans. Later, or probably during the same time, there was the emergence of an infectious disease called "plague", which swept across many countries and caused a lot of mortality. This disease was also called the black death, due to the nature of lesions caused and the thousands of people who were dying in very less time. We have also seen the emergence of a novel influenza virus, the Spanish flu (1918) which caused a severe pandemic. Interestingly, all these infectious diseases caused pandemics involving several countries, and causing increased mortality, especially in the European continent. In this editorial I discuss the significance of the most recent pandemic caused by the Severe Acute Respiratory Syndrome-Coronavirus-2 (SARS-CoV2), also called Coronavirus disease-19 (COVID-19).

Key words: Severe Acute Respiratory Syndrome-Coronavirus2 (SARS-CoV-2), COVID-19, emergence, re-emergence, infectious diseases, Pandemic

\section{Introduction}

The recent emergence of a novel Coronavirus, now named as Severe Acute Respiratory Syndrome-Coronavirus-2 (SARS-CoV-2), is just another microbial species that have been added new to the list of notorious microbes which had emerged to infect the humans for the first time [1]. The SARS-CoV-2 belongs to the family of CoVs, which have been known to exist both in the animals and humans. Few CoVs are responsible for the frequent and seasonal episodes of cold in humans.

The CoVs, because of their wide host adaptability have been able to establish themselves in the environment. Currently, there are seven recognized human CoVs (HCoVs) that have been associated with human infections. Barring the SARSCoV (emerged in China, caused pandemic from 2002-2003), and the Middle East Respiratory Syndrome-CoV (emerged in Saudi Arabia, caused a pandemic in 2012), and the SARS-CoV2 (2019-?), which resulted in severe and life-threatening infections, other HCoVs have only been associated with mild and self-limiting infections in humans.

The emergence and re-emergence of microbial species are not new to the world as evidenced by our previous experiences with the Influenza virus, the Chikungunya virus, the Dengue virus, the Ebola virus, the Zika virus, and many others. Most emergences of novel microbial species have been associated with the ability of the microbe to survive in different animal species and to undergo genetic recombination. The emergence of novel microbial species might also happen when some microbes which exclusively cause animal infections, spill into the humans either by accident or by abnormal human behaviors, which include moving into the wild, rearing exotic animals, and consuming wild animals' meat. Interestingly, most microbial emergences in the last two decades have had a zoonotic source and include SARS-CoV-2 (Bats/Pangolins/other animals), SARS-CoV (Bats/civet cats, and other animals), MERSCoV (dromedary Camels), , Influenza (birds, swine, and other animals), Ebola (fruit bats, monkeys, and other wild animals), and Zika virus (monkeys, Aedes mosquito). The potential mechanism behind the emergence of novel microbial species is shown in 


\section{Editorial}

Figure 1
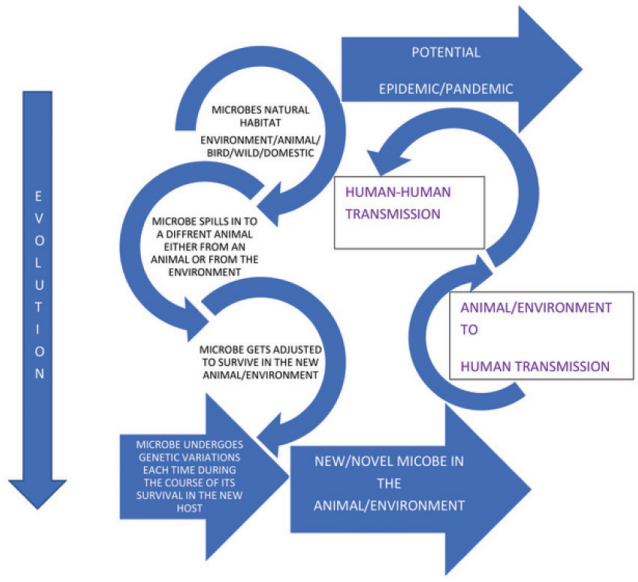

Figure 1: Potential mechanism behind the evolution of a novel microbe/microbial species ${ }^{4}$.

\section{The emergence and significance of SARS-CoV-2}

The SARS-CoV-2 which has emerged in 2019, in the Wuhan city of China, has been noted to have more than $90 \%$ similarity to the Bat CoVs, and Pangolin CoV. What differentiates SARSCoV-2 with them is the protein moieties, and the ability of the newly emerged virus to bind effectively to the Angiotensinconverting enzyme-2 (ACE2) receptors, which are present on various cells of the humans including the respiratory cells with the help of a receptor-binding enzyme (RBE), the transmembrane protease serine 2 (TMPRSS2) [2].

What separates SARS-CoV-2 from other emergent microorganisms is its ability to establish itself in the human cells, cause asymptomatic, and mild to severe and lifethreatening infections. Also, it is now established that the SARSCoV-2 spreads fast and has a high RO (R naught, a mathematical representation of the ability of a microbe to transmit the infection) (>5 and up to ? ), as compared to its predecessors SARS-CoV $(R 0<1)$, and MERS-CoV $(R 0<1)$. The R0 defines the contagious nature of a microbe, and it correlates directly with the number of people who can be infected by a single infected person. The RO of the SARS-CoV-2 appears to be either equal or more than the 1918 Spanish flue (R0 >1 and up to 2.4) and the 2009 Influenza virus ( $R 0>2$, and up to 16 ). The R0 is inversely proportional to the doubling time and a rise in the Ro will reduce the doubling time of the infections, hospitalizations, and deaths due to infections. The doubling time for infection with SARS-CoV-2 is variable throughout the world and ranges from as low as 2 days (the United States of America, Spain, Italy) and as high as 6 days (India) in the initial phases of the pandemic.

\section{Clinical features and diagnosis of COVID-19}

The people infected with SARS-CoV-2, after an incubation period (IP) of 3-6 days (minimum and maximum IP depends on the virulence of the infection causing strain/variant and the immune status of the person) may present with fever, sore throat, dry cough, and difficulty of breathing. The difficulty of breathing appears to be a prominent symptom that augurs suspicion of COVID-19.

Preliminary diagnosis of COVID-19 depends majorly on the signs and symptoms of patients on presentation, history of travel, contact with a positive/suspected person. Laboratory diagnosis and confirmation of infection are carried out by detecting the nucleic acid in the nasal/oral secretions, and throat swab using various nucleic acid amplification techniques that include the real-time Polymerase Chain Reaction (rt-PCR), Reverse Transcription PCR (RT-PCR), and Loop-mediated isothermal amplification (LAMP) based techniques. Detection of antibodies in the patient's blood using the enzyme-linked immunosorbent assay (ELISA) may also be used, but its efficacy in laboratory diagnosis is still in a debate because not always antibodies against the SARS-CoV-2 are detected.

Because of the cost associated with the testing and confirmation of the disease, due to the severity of the disease transmission, and the morbidity and the mortality associated with the infection, what best we can do in a pandemic like the situation that is currently present is to follow social distancing, use protective face masks, and avoid social gatherings. The healthcare professionals are the first line personnel who deal with the suspected/positive cases must make sure to use personal protective equipment, and other measures to prevent the infection.

\section{Therapeutics and management of COVID-19}

Another problem with COVID-19 is the unavailability of a specific antiviral drug, and vaccine to prevent the infection. This makes it more difficult to stop the spread of transmission and treat/manage the infected people. A few candidate drugs (under clinical trial) like the angiotensin-converting enzyme 2 (ACE2) inhibitors, transmembrane protease/serine subfamily member 2 (TMPRSS2) inhibitors, FoipanTM/camostat mesylate and several repurposed drugs (chloroquine and hydroxychloroquine, remdesivir, lopinavir, ritonavir, arbidol, and favipiravir) are currently used to treat and manage the infected patients [3].

The SARS-CoV-2 is equally infectious to all the age groups, but studies have noted that males are more infected as compared to females. This, probably because males are the ones who venture out of the houses very frequently. Most (up to $80 \%$ ) infections remain self-limiting and treatment might be required in the rest of the cases, with $<5 \%$ requiring mechanical ventilation. Immunosuppression due to age (less than 10 years and $>65$ years), presence of co-morbid conditions like the cardiovascular, respiratory, and metabolic disorders, chronic kidney diseases may predispose to complications of COVID19. 
It appears that the virus has spread to most parts of the world infecting more than 3.7 million people and killing more than 250,000 people globally. Studies thus far have found the evidence regarding the genetic variations of the virus. There are contrasting reports of the number of circulating SARS-CoV2 strains currently found throughout the world. Also, there are some reports which suggested that the infection and mortality rate variations observed in different countries may be attributed to the SARS-CoV-2 variant/strain circulating in that geographical region.

\section{Current perspectives}

From the available literature, we can predict many possibilities regarding the course of this pandemic and the SARS-CoV-2. The novel virus (SARS-CoV-2) which emerged from the Chinese mainland in the year 2019 and had spread throughout the world may in due course of time undergo genetic variations and become less virulent and could establish itself in the environment for years infecting humans and animals and causing mild to self-limiting respiratory tract infections similar to the frequently re-emerging Influenza virus.

The virus may undergo significant genetic modifications and could get eliminated from the humans as we have seen with the case of SARS-CoV (2002-2003), which remained almost invisible after July 2003, except for a couple of laboratory-leak linked re-emergences and a re-emergence in Southern China (suspected animal-human transmission). The MERS-CoV outbreak, which emerged as a novel Coronavirus in 2012, and caused human infections in the Middle East countries including the Kingdom of Saudi Arabia, Qatar, and others, although had not spread throughout the world, it is still a potential viral infection which can happen in people living closely with Camels, and the travelers to these geographical regions.

Scientists throughout the world have started working on a specific therapeutic agent and a vaccine against SARS-CoV-2, and time only can tell us about its availability.

Interestingly, there is still no reliable drug or a vaccine against several viral infections like Influenza, Dengue, Chikungunya, SARS-CoV, MERS-CoV, and many others. Re-emergences of these viruses may pose a potential public health threat anytime in the future.

\section{Conclusion}

Therefore, people should minimize intra-country and intercountry travel until the virus spread comes to a halt, or people develop herd immunity. Also, important is the strict cough etiquette, use of masks in public places, following the best hand hygiene practices to control and prevent the spread of infection until a drug and an approved vaccine is available.

\section{REFERENCES}

1. Pal M, Berhanu G, Desalegn C, Kandi V. Severe Acute Respiratory Syndrome Coronavirus-2 (SARS-CoV-2): An Update. Cureus. 2020;12(3): e7423. Published 2020 Mar 26. doi:10.7759/cureus.7423

2. Verdecchia P, Cavallini C, Spanevello A, Angeli F. The pivotal link between ACE2 deficiency and SARS-CoV-2 infection [published online ahead of print, $2020 \mathrm{Apr} 20$ ]. Eur J Intern Med. 2020; S0953-6205(20)30151-5. doi: 10.1016/ j.ejim.2020.04.037

3. McKee DL, Sternberg A, Stange U, Laufer S, Naujokat C. Candidate drugs against SARS-CoV-2 and COVID-19 [published online ahead of print, 2020 Apr 29]. Pharmacol Res. 2020;104859. doi: 10.1016/j.phrs.2020.104859

4. Pal M, Kerorosa GB, Kandi V. Aknowledge update on SARSCoronavirus-2 (SARS-COV-2) / Covid-19 and it's global public health implications. American Journal of Clinical Medicine Research 2020;8:23-27. doi: 10.12691/ajcmr-8$1-5$

How to cite this article : Kandi V. Coronavirus Disease (COVID-19)/SARS-CoV-2: Hopefully, the Human-Virus Battle Ends Soon on a Positive Note. Perspectives in Medical Research 2020; 8(1): 1-3

Sources of Support: Nil, Conflict of interest: None declared 